\title{
Jejunojejunal Intussusception following Jejunostomy
}

\author{
Younes Aggouri*, Karim Ibn Majdoub, Issam Yazough, Mourad Oussaid, \\ Imane Tourghai, Said Ait Laalim, Khalid Mazaz \\ Département de Chirurgie Générale (B), CHU Hassan II, Fès, Maroc \\ Email: draggouriyou@gmail.com
}

Received 23 March 2015; accepted 17 April 2015; published 22 April 2015

Copyright (C) 2015 by authors and Scientific Research Publishing Inc.

This work is licensed under the Creative Commons Attribution International License (CC BY).

http://creativecommons.org/licenses/by/4.0/

cC) (i) Open Access

\begin{abstract}
Intussusception is an invagination of a segment of the gastrointestinal tract into an adjacent one. Jejunojejunal intussusception is a rare complication of jejunostomy tube placement with an incidence of $1 \%$. We are reporting a case of 35-year-old man who was suffering from severe oral, lesions due to Ingestion of acide agents. He received Witzel jejunostomy for early feeding. Ileus developed postoperatively and plain $\mathrm{X}$-ray of the abdomen showed distended small bowel loop. Abdominal computed tomography revealed target sign as well as the feeding tube in a dilated jejunum and intussusception was diagnosed. Exploratory laparotomy was required due to failure of expectant therapy. Reduction of intussusception was done during exploratory laparotomy. The jejunostomy feeding was continued and the postoperative course was uneventful.
\end{abstract}

\section{Keywords}

Intussusception, Jejunostomy, Invagination

\section{Introduction}

Intussusception is an invagination of a segment of the gastrointestinal tract into an adjacent one. Jejunojejunal intussusception is a rare complication of jejunostomy tube placement [1]. We describe a new case with jejunojejunal intussusceptions induced by jejunostomy tube placement requiring an operative treatment. Reduction of intussusception was performed, followed by an uneventful recovery; tube feeding was continuously retained through the previous jejunostomy tube.

\section{Case Report}

A 35 year-old man presented to an outside institution for ingestion of acid agent, intentionality of the ingestion

${ }^{*}$ Corresponding author.

How to cite this paper: Aggouri, Y., Ibn Majdoub, K., Yazough, I., Oussaid, M., Tourghai, I., Laalim, S.A. and Mazaz, K. (2015) Jejunojejunal Intussusception following Jejunostomy. Surgical Science, 6, 175-178. 
was suicidal, esophagogastroduodenoscopy between H12 and H24 findings IIB esophagus and III A stomach to Zargar classification, with severe oral lesions. Witzel jejunostomy was done to allow early feeding. The patient was recovering well after adequate nutritional support via jejunostomy tube feeding; however, vomiting occurred on the 5th postoperative day. After vomiting, colicky pain was relieved, and vomiting continues despite the antiemetic treatment. Plain abdominal X-ray showed a dilated small bowel loop in the left upper abdomen, Due to the persistent intermittent symptoms a CT was performed, Abdominal CT identified a target sign in the left abdomen, and intussusception was diagnosed (Figure 1), in our case abdominal US was not performed because initially we don't suspect the jejunojejunal intussusception.

Therefore, and after patient consent, an exploratory laparotomy was performed due to failure of expectant therapy, and a $10 \mathrm{~cm}$ long fleshy sausage-like tubular intestinal mass was found at the proximal jejunum, $15 \mathrm{~cm}$ distal to the entry of the jejunostomy tube (Figure 2). The color of the bowel was pink and normal, the intussusception was antegrade in direction, and the jejunostomy tube was in a normal position, there were 2 others intussusceptions distally (Figure 3). Complete and careful reduction of the intussusceptions was performed without any change to the previous jejunostomy tube. Postoperative recovery was uneventful, and the patient was discharged 1 week later. On follow-up the feeding jejunostomy was functioning well and patient had gained weight after four weeks.

\section{Discussion}

Jejunostomy is a surgical procedure by which a tube is situated in the lumen of the proximal jejunum, primarily to administer nutrition [1]. Enteral feeding is the most preferable route for nutritional support due to its trophic effects on the gastrointestinal tract causing less bacterial translocation and low infective complications when compared with the parenteral route [2]. There are many techniques used for jejunostomy, including longitudinal Witzel, transverse Witzel, open gastrojejunostomy, needle catheter technique, percutaneous endoscopy, and laparoscopy [2] [3].

The common complications of tube jejunostomy described in literature are mechanical (tube dislocation, ob-

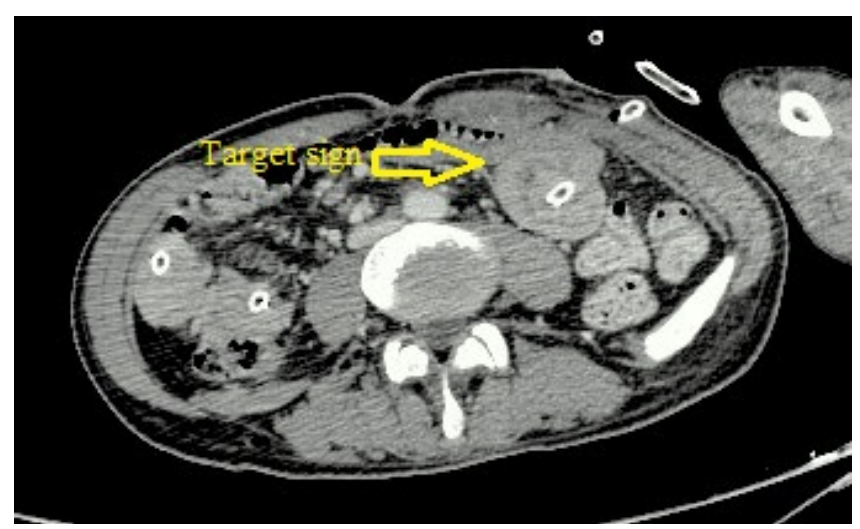

Figure 1. Abdominal computed tomography shows a target sign with a jejunostomy tube in the center.

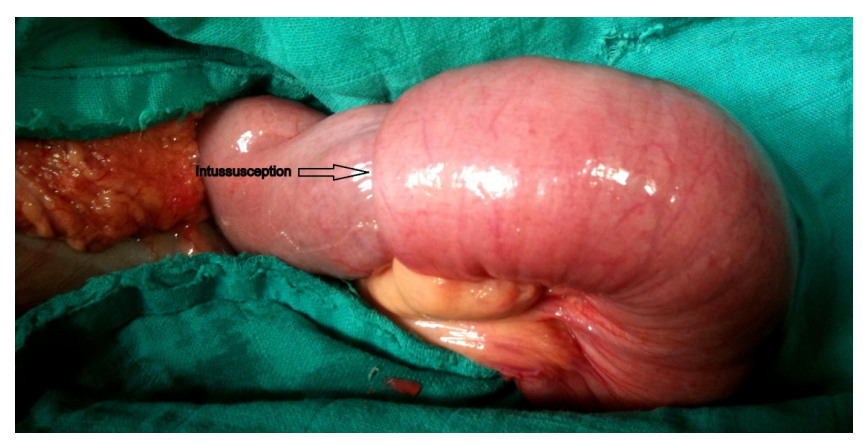

Figure 2. Proximal jejuno-jejunal intussusception. 


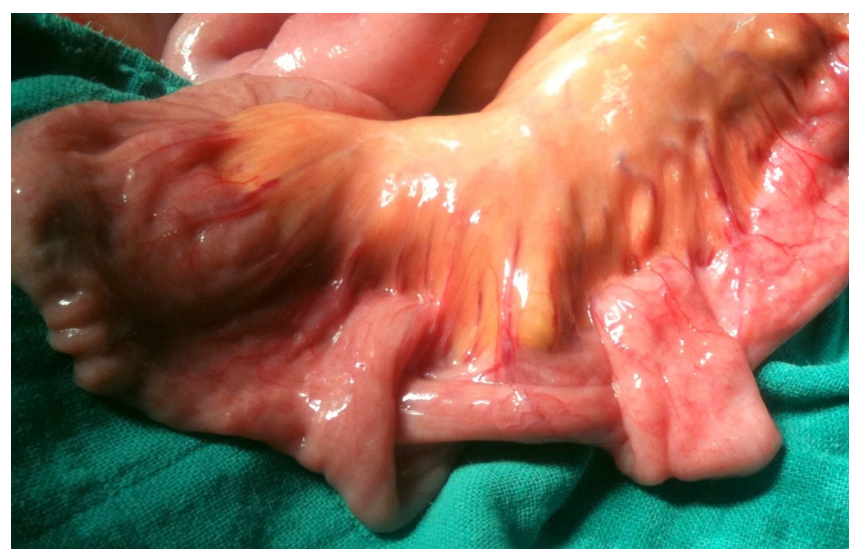

Figure 3. Others reducible jejuno-jejunal intussusceptions distal to the primary one.

struction or migration), infectious (cutaneous or intra-abdominal abscesses, aspiration pneumonia, peritonitis), gastrointestinal and metabolic abnormalities (hyperglycemia, hypokalemia, water and electrolyte imbalance, hypophosphatemia, hypomagnesemia) [1] [3]. These may be minor or major requiring medical or surgical interventions. These complications can be early ( $<30$ days) or late ( $>30$ days) [3]. Major complications are smallbowel narrowing, extra luminal tracks or collections, jejunal haematomas and focal intussusception of the small bowel at the site of the jejunostomy tube [4]. Jejunostomy tube-induced intussusception is rare with an incidence of 1\% [2] [4]. Intussusception may be transient or intermittent. Common presentations are bilious vomiting, pain and sometimes transient mass per abdomen or may be entirely asymptomatic. Predisposing factors may be men, young infants and the presence of distal pigtail on the tube [2] [4].

Jejunojejunal intussusception induced by jejunostomy tube was first reported in a series of four patients. The four patients had no clinical symptoms found at radiographic examination, and the intussusception resolved without intervention [1] [4]. However, the need for operative management as in the present case has been previously reported in only 2 cases [1] [2] [5]; first case require resection of the involved segment of small intestine, bowel continuity was maintained with a jejuno-jejunal anastomosis and a new feeding jejunostomy was placed $15 \mathrm{~cm}$ distal to the anastomotic site [2]. The treatment of the second case was similar at our patient [1].

The diagnosis of jejunostomy tube-induced intussusceptions is difficult as it may not disturb with tube feeding. Usually diagnosis is arrived on the basis of clinical symptoms and sonography or tomographic scans [5]. The mechanism of jejunostomy tube-induced intussusceptions is not known. Couple of theories has been proposed. It may be due to the tip of the tube acting as a lead point and dragging of the bowel; tube-induced inflammatory reaction causing hypertrophy of the mucosa which forms the lead point; retrograde peristalsis of jejunum due to vomiting and injecting force during feeding using an infusion pump which also acts as a lead point. Poorly built patients have reduced fatty tissue (omentum, mesentery), allows free movement of intestine which also is an added risk factor [1] [5]. In our case the intussusception was away from the point of entry and also not near the tip of the tube.

Jejunostomy tube-induced intussusception usually resolves spontaneously but also may require operative intervention. Usually tube-induced intussusceptions are managed by changing the tube to standard or short tube without distal pigtail [2] [6], or reduction by injection of air or contrast via the tube or exchange over a wire under fluoroscopic guidance. Radiological reduction by air enema or contrast medium provides uncertain results [6].

Our patient had obstructive gastrointestinal symptoms, Exploratory laparotomy was required due to failure of expectant therapy. Jejunojejunal intussusception without gangrenous change was identified during the operation, and the jejunostomy tube was still in the normal position. The patient was successfully treated with operative reduction without resection of the small intestine, and he recovered well postoperatively. Although the jejunostomy tube had caused the intussusception, it was not removed during the operation, and no resection of the intussusception was done. This suggests that evacuation of the jejunostomy and resection of the bowel segment of intussusceptions to prevent recurrence may not be necessary except in the case when intussusception was not 
reducible, gangrenous change, perforation or stenosis. [1] [2] [5].

\section{Conclusion}

Feeding jejunostomy is usually not associated with major complications requiring surgery. Those patients who are having persistent pain and vomiting, a diagnosis of intussusception should be considered, even though rare. Operative treatment will be required in patients having the above persistent symptoms thereby reducing the morbidity and suffering.

\section{Conflicts of Interest}

The authors report no conflicts of interest.

\section{References}

[1] Wu, T.H., Lin, C.W. and Yin, W.Y. (2006) Jejunojejunal Intussusception Following Jejunostomy. Journal of the Formosan Medical Association, 105, 355-358. http://dx.doi.org/10.1016/S0929-6646(09)60129-7

[2] Krishna, S., Prabhu, R., Thangavelu, S. and Shenoy, R. (2013) Jejuno-Jejunal Intussusception: An Unusual Complication of Feeding Jejunostomy. BMJ Case Reports. http://dx.doi.org/10.1136/bcr-2013-200219

[3] Friedman, J.N., Ahmed, S., Connolly, B., Chait, P. and Mahant, S. (2004) Complications Associated with ImageGuided Gastrostomy and Gastrojejunostomy Tubes in Children. Pediatrics, 114, 458-461. http://dx.doi.org/10.1542/peds.114.2.458

[4] Carucci, L.R., Levine, M.S., Rubesin, S.E., Laufer, I., Assad, S. and Herlinger, H. (2002) Evaluation of Patients with Jejunostomy Tubes: Imaging Findings. Radiology, 223, 241-247. http://dx.doi.org/10.1148/radiol.2231010961

[5] Connolly, B.L., Chait, P.G., Siva-Nandan, R., Duncan, D. and Peer, M. (1998) Recognition of Intussusceptions around Gastrojejunostomy Tubes in Children. American Journal of Roentgenology, 170, 467-470. http://dx.doi.org/10.2214/ajr.170.2.9456966

[6] Parikh, M., Samujh, R., Kanojia, R. and Sodhi, K.S. (2010) Does All Small Bowel Intussusception Need Exploration. African Journal of Paediatric Surgery, 7, 30-32. http://dx.doi.org/10.4103/0189-6725.59358 